\title{
SYMPLECTIC BILINEAR FORMS ON AFFINE REAL ALGEBRAIC SURFACES
}

\author{
by W. KUCHARZ
}

(Received 17 February, 1988)

1. Introduction. Given a commutative ring $A$ with identity, let $W^{-1}(A)$ denote the Witt group of skew-symmetric bilinear forms over $A$ (cf. [1] or [7] for the definition of $\left.W^{-1}(A)\right)$.

For a regular affine 2-dimensional algebra $A$ over the field $\mathbf{R}$ of real numbers, the group $W^{-1}(A)$ has been computed in a quite explicit way in [1] under the assumption that the set $X(\mathbf{R})$ of $\mathbf{R}$-rational points of $X=\operatorname{Spec} A$ is compact in the strong topology (induced by the Euclidean topology on $\mathbf{R}$ ). In fact the calculation of $W^{-1}(A)$ has been reduced to the calculation of the subgroup $H_{\mathrm{alg}}^{1}(X(\mathbf{R}), \mathbf{Z} / 2)$ of the cohomology group $H^{1}(X(\mathbf{R}), \mathbf{Z} / 2)$ generated by the cohomology classes of algebraic cycles on $X$ of codimension 1 (cf. Section 2 for the definition of $H_{\mathrm{alg}}^{1}(X(\mathbf{R}), \mathbf{Z} / 2)$ ). This result of [1] does not seem directly to extend to the case with $X(\mathbf{R})$ not necessarily compact. Here, without any restrictions on $X(\mathbf{R})$, we show that the group $W^{-1}(A) / 2 W^{-1}(A)$ is canonically isomorphic to the quotient group of $H^{2}(X(\mathbf{R}), \mathbf{Z} / 2)$ modulo the subgroup

$$
\left\{v^{2}=v \cup v \in H^{2}(X(\mathbf{R}), \mathbf{Z} / 2) \mid v \in H_{\text {alg }}^{1}(X(\mathbf{R}), \mathbf{Z} / 2)\right\} .
$$

In particular, if $X(\mathbf{R})$ has no compact connected component then $H^{2}(X(\mathbf{R}), \mathbf{Z} / 2)=0$ and hence $W^{-1}(A) / 2 W^{-1}(A)=0$.

For more information about $H_{\mathrm{alg}}^{1}(X(\mathbf{R}), \mathbf{Z} / 2)$, we refer to [2] and [8].

2. Results. First we need some preparation. Let $X$ be a smooth quasi-projective variety over $\mathbf{R}$. The set $X(\mathbf{R})$ of $\mathbf{R}$-rational points of $X$, equipped with the strong topology, has the natural structure of a $C^{\infty}$ manifold, $X(\mathbf{R})=\varnothing$ or $\operatorname{dim} X(\mathbf{R})=\operatorname{dim} X$. Given a closed $d$-dimensional subvariety $Y$ of $X$, let $|Y|$ denote the fundamental class of $Y(\mathbf{R})$ in $H_{d}^{B M}(Y(\mathbf{R}), \mathbf{Z} / 2)$ (cf. [3, 5.12]; if $\operatorname{dim} Y(\mathbf{R})<d$, we set $\left.|Y|=0\right)$, where $H_{*}^{B M}(\cdot, \mathbf{Z} / 2)$ stands for the Borel-Moore homology with coefficients in $Z / 2$. Let

$$
i_{Y}: H_{*}^{B M}(Y(\mathbf{R}), \mathbf{Z} / 2) \rightarrow H_{*}^{B M}(X(\mathbf{R}), \mathbf{Z} / 2)
$$

be the homomorphism induced by the inclusion $Y(\mathbf{R}) \subset X(\mathbf{R})$ and let

$$
\Delta: H_{*}^{B M}(X(\mathbf{R}), \mathbf{Z} / 2) \rightarrow H^{*}(X(\mathbf{R}), \mathbf{Z} / 2)
$$

be the Poincare duality isomorphism, where $H^{*}(\cdot, \mathbf{Z} / 2)$ is the cohomology with coefficients in the constant sheaf $\mathbf{Z} / 2$ (which in the case considered here is the same as the singular cohomology). There is a unique homomorphism of graded rings

$$
\mathrm{cl}_{X}: A(X) \rightarrow H^{*}(X(\mathbf{R}), \mathbf{Z} / 2)
$$

such that $\mathrm{cl}_{X}([Y])=\Delta\left(i_{Y}(|Y|)\right)$ for all closed subvarieties $Y$ of $X$, where $A(X)$ is the Chow ring of $X$ and $[Y]$ is the cycle represented by $Y$ (cf. [3, 5.13]). We set

$$
H_{\mathrm{alg}}^{*}(X(\mathbf{R}), \mathbf{Z} / 2)=\underset{k \geq 0}{\bigoplus} H_{\mathrm{alg}}^{k}(X(\mathbf{R}), \mathbf{Z} / 2)=\mathrm{cl}_{X}(A(X)) .
$$

Glasgow Math. J. 31 (1989) 195-198. 
Note that

$$
G(X(\mathbf{R}))=\left\{v^{2} \in H^{2}(X(\mathbf{R}), \mathbf{Z} / 2) \mid v \in H_{\mathrm{alg}}^{1}(X(\mathbf{R}), \mathbf{Z} / 2)\right\}
$$

is a subgroup of $H^{2}(X(\mathbf{R}), \mathbf{Z} / 2)$.

Theorem 1. Let $A$ be a regular affine $\mathbf{R}$-algebra of dimension 2 and let $X=\operatorname{Spec} A$. Then the groups $W^{-1}(A) / 2 W^{-1}(A)$ and $H^{2}(X(\mathbf{R}), \mathbf{Z} / 2) / G(X(\mathbf{R}))$ are canonically isomorphic. Moreover, $W^{-1}(A)=0$ if $X(\mathbf{R})$ is empty.

In particular, we immediately obtain the following.

Corollary 2. Let $A$ be a regular affine $\mathbf{R}$-algebra of dimension 2 and let $X=\operatorname{Spec} A$. If $X(\mathbf{R})$ is nonempty and the $C^{\infty}$ surface $X(\mathbf{R})$ has no compact connected nonorientable component, then $W^{-1}(A) / 2 W^{-1}(A)$ is isomorphic to $(\mathbf{Z} / 2)^{s}$, where $s$ is the number of compact connected components of $X(\mathbf{R})$.

3. Proof of Theorem 1. Let $A$ be a commutative ring with identity. Denote by $K_{0}(A)$ the Grothendieck group of finitely generated projective $A$-modules and by $\operatorname{Pic}(A)$ the Picard group of $A$, and let $S K_{0}(A)$ be the kernel of the determinant homomorphism $\operatorname{det}: K_{0}(A) \rightarrow \operatorname{Pic}(A)$. One easily sees that the subgroup $\operatorname{tr} \tilde{K}_{0}(A)$ of $K_{0}(A)$ generated by the classes of all elements of the form $P \oplus P^{*}$, where $P$ is a finitely generated projective $A$-module and $P^{*}$ is the dual module, is contained in $S K_{0}(A)$. We shall be using the main result of [7].

THEOREM 3 [7]. Let $A$ be a 2-dimensional affine algebra over a real closed field $R$. Then the groups $W^{-1}(A) / 2 W^{-1}(A)$ and $S K_{0}(A) / \operatorname{tr} \tilde{K}_{0}(A)$ are canonically isomorphic. Moreover, if the set of $R$-rational points of $\operatorname{Spec} A$ is contained in a closed subscheme of dimension not exceeding 1 , then the groups $W^{-1}(A)$ and $S K_{0}(A) / \operatorname{tr} \tilde{K}_{0}(A)$ are canonically isomorphic.

Proof of Theorem 1. Without loss of generality, we may assume that $X$ is irreducible. We shall identify finitely generated projective $A$-modules with vector bundles on $X$. Given a vector bundle $E$ on $X$, let $c_{i}(E)$ denote its $i$ th Chern class with value in the Chow group $A^{i}(X)$ of $X$. We also consider $c_{i}$ as a mapping from $K_{0}(A)$ to $A^{i}(X)$ and denote by $[E]$ the class in $K_{0}(A)$ represented by $E$.

The group homomorphism

$$
\varphi: S K_{0}(A) \rightarrow H^{2}(X(R), \mathbf{Z} / 2),
$$

induced by the mapping which associates to every vector bundle $E$ on $X$ the element $\mathrm{cl}_{X}\left(c_{2}(E)\right)$, satisfies $\varphi\left(\operatorname{tr} \bar{K}_{0}(A)\right) \subset G(X(R))$ and hence gives rise to the group homomorphism

$$
\psi: S K_{0}(A) / \operatorname{tr} \tilde{K}_{0}(A) \rightarrow H^{2}(X(R), \mathbf{Z} / 2) / G(X(R)) .
$$

We claim that $\psi$ is a bijection.

Observe that $H^{2}(X(R), \mathbf{Z} / 2)=H_{\mathrm{alg}}^{2}(X(R), \mathbf{Z} / 2)$ since $\operatorname{dim} X=2$. Thus surjectivity of 
$\psi$ follows immediately from the fact that $c_{2}: S K_{0}(A) \rightarrow A^{2}(X)$ is an isomorphism (cf. [5, Example 15.3.6]).

It remains to show that $\psi$ is injective. First let us observe that

$$
\Gamma=\left\{a \in A^{2}(X) \mid \mathrm{cl}_{X}(a)=0\right\}
$$

is a divisible subgroup of $A^{2}(X)$. Indeed, by Hironaka's theorem [6], we may assume that $X$ is an open subvariety of a projective nonsingular algebraic surface $Y$ over $\mathbf{R}$. Consider the following commutative diagram

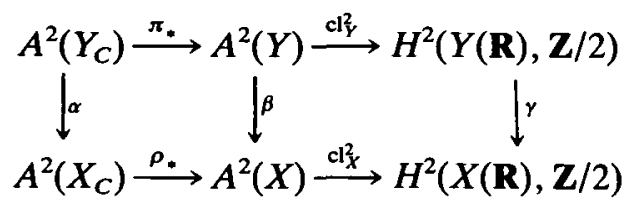

where $Y_{\mathbf{C}}=Y \times_{\mathbf{R}} \mathbf{C}, X_{\mathbf{C}}=X \times_{\mathbf{R}} \mathbf{C}$, the homomorphisms $\pi_{*}, \rho_{*}$ are induced by the canonical projections $\pi: Y_{\mathbf{C}} \rightarrow Y, \rho=\pi \mid X_{\mathbf{C}}: X_{\mathbf{C}} \rightarrow X$, the vertical arrows are the homomorphisms induced by the inclusions $X_{\mathbf{C}} \subset Y_{\mathbf{C}}, X \subset Y, X(\mathbf{R}) \subset Y(\mathbf{R})$, and the homomorphisms $\mathrm{cl}_{Y}^{2}, \mathrm{cl}_{X}^{2}$ are the restrictions of $\mathrm{cl}_{Y}, \mathrm{cl}_{X}$. By [4], the top row of the diagram is exact. We shall demonstrate that the bottom row is exact too. Obviously, $\mathrm{cl}_{X}^{2}{ }^{\circ} \rho_{*}=0$. Let $\mathrm{cl}_{X}^{2}(u)=0$ for some $u$ in $A^{2}(X)$ and let $u=\beta(v)$ for some $v$ in $A^{2}(Y)$. Since $\gamma\left(\mathrm{cl}_{Y}^{2}(v)\right)=0$, one can find an element $w$ in $A^{2}(Y)$, represented by a $Z$-linear combination of points of $Y(\mathbf{R}) \backslash X(\mathbf{R})$, such that $\mathrm{cl}_{Y}^{2}(v-w)=0$. By construction, $\beta(v-w)=0$ and hence exactness of the top row implies exactness of the bottom row of the diagram.

Let $\tilde{A}^{2}\left(Y_{\mathbf{C}}\right)$ be the subgroup of $A^{2}\left(Y_{\mathbf{C}}\right)$ of 0 -cycles (here $\left.\operatorname{dim} Y_{\mathrm{C}}=2\right)$ of degree zero. Thus $\tilde{A}^{2}\left(Y_{\mathbf{C}}\right)$ is a divisible group (cf. [5, Example 1.6.6]). Since $X_{\mathbf{C}}$ is an open affine subvariety of $X_{\mathbf{C}}$, it follows that $\alpha\left(\tilde{A}^{2}\left(Y_{\mathbf{C}}\right)\right)=A^{2}\left(X_{\mathbf{C}}\right)$, and hence $A^{2}\left(X_{\mathbf{C}}\right)$ is a divisible group. Therefore, $\operatorname{im}\left(\rho_{*}\right)=\operatorname{ker}\left(\mathrm{cl}_{X}^{2}\right)=\Gamma$ is a divisible group.

Now we return to the proof of injectivity of $\psi$. Let $\xi$ be an element of $S K_{0}(A)$ satisfying $\mathrm{cl}_{X}\left(c_{2}(\xi)\right)=v^{2}$ for some $v$ in $H_{\text {alg }}^{1}(X(R), \mathbf{Z} / 2)$. Pick a line bundle $L$ such that $\operatorname{cl}_{X}\left(c_{1}(L)\right)=v$. Then, for $\eta=\xi+\left[L \oplus L^{*}\right]$, we obtain, $c_{1}(\eta)=0$ and $c_{2}(\eta)=a$, where $a$ is in $\Gamma$. Choose an element $b$ in $\Gamma$ satisfying $2 b=-a$. By [5, Example 15.3.6], there exists a vector bundle $F$ on $X$ such that $c_{1}(F)=0$ and $c_{2}(F)=b$. Clearly, $c_{1}\left(F^{*}\right)=0$ and $c_{2}\left(F^{*}\right)=b$ and hence setting $\zeta=\eta+\left[F \oplus F^{*}\right]$, we obtain $c_{1}(\zeta)=0$ and $c_{2}(\zeta)=0$. Applying [5, Example 15.3.6] once again, we conclude that $\xi=0$ and hence $\xi$ belongs to $\operatorname{tr} \bar{K}_{0}(A)$. This shows that $\psi$ is injective.

In view of Theorem 3, the proof of Theorem 1 is complete.

\section{REFERENCES}

1. J. Barge and M. Ojanguren, Fibrés algébriques sur une surface réelle, Comment. Math. Helv. 62 (1987), 616-629.

2. J. Bochnak and W. Kucharz, Sur les classes d'homologie représentables par des hypersurfaces algébriques réelles, C. R. Acad. Sci. Paris Sér. I Math. 305 (1987), 609-611. 
3. A. Borel and A. Haefliger, La classe d'homologie fondamentale d'un espace analytique, Bull. Soc. Math. France 89 (1961), 461-513.

4. J.-L. Colliet-Thélène and F. Ischebeck, L'èquivalence rationelle sur les cycles de dimension zéro des variétés algébriques réelles, C. R. Acad. Sci. Paris Sér. I Math. 292 (1981), 723-725.

5. W. Fulton, Intersection Theory, Ergebnisse der Math. vol. 2 (Springer, 1984).

6. H. Hironaka, Resolution of singularities of an algebraic variety over a field of characteristic zero I, II, Ann. of Math. (2) 79 (1964), 109-326.

7. M. Ojanguren, R. Parimala and R. Sridharan, Symplectic bundles over affine surfaces, Comment. Math. Helv. 61 (1986), 491-500.

8. R. Silhol, Cohomologie de Galois et cohomologie des variétés algébriques réelles; applications aux surfaces rationelles, Bull. Soc. Math. France 115 (1987), 107-125.

Department of Mathematics and Statistics

UNIVERSITY OF NEW MEXICO

Albuqueroue, New MeXico 87131 